\title{
LOSS OF TUMOR NECROSIS FACTOR PRODUCTION BY HUMAN MONOCYTES IN FALCIPARUM MALARIA AFTER THEIR MATURATION IN VITRO
}

\author{
TAKAYOSHI NAGAO, HARUKI UEMURA, TETSUO YANAGI, KAZUNORI OISHI, \\ TSUYOSHI NAGATAKE, AND HIROJI KANBARA \\ Department of Protozoology, and Department of Internal Medicine, Institute of Tropical Medicine, \\ Nagasaki University, Nagasaki, Japan
}

\begin{abstract}
In Plasmodium-infected mammals, phagocytosis and production of tumor necrosis factor (TNF) by monocytes and macrophages are prominent features. The present work aimed at clarifying the relationship between the maturation of human monocytes to macrophages and their TNF productivity and phagocytic ability in the presence of Plasmodium falciparum-infected erythrocytes. Fresh monocytes produced a significantly higher quantity of TNF in the presence of schizont-infected erythrocytes than macrophages obtained by in vitro monocyte maturation on autologous serum, whereas phagocytic activity of macrophages was much higher than that of fresh monocytes. This indicated that the TNF-inducing factors from $P$. falciparum-infected erythrocytes could stimulate fresh monocytes, but not macrophages, to release TNF, regardless of their development of phagocytosis. Activation of macrophages by interferon- $\gamma$ could not recover their TNF productivity in the presence of $\boldsymbol{P}$. falciparum-infected erythrocytes, but it enhanced their TNF productivity in the presence of lipopolysaccharide(s). The TNF-inducing factors were contained mainly in erythrocytes infected with mature schizonts but not in erythrocytes infected with the younger stages of the parasites. Fractionation of infected erythrocytes revealed that both soluble and insoluble components almost equally contained those factors.
\end{abstract}

Plasmodium infection has been shown to be one of the typical infectious diseases that show increased serum levels of tumor necrosis factor (TNF). ${ }^{1,2}$ Although TNF plays an important role in host resistance to malaria parasites, ${ }^{3-6}$ excessive TNF production seems to be implicated in severe complications of Plasmodium falciparum and $P$. berghei malaria. ${ }^{7-11}$ Soluble substances released from infected erythrocytes at schizont rupture have been shown to stimulate macrophages directly to produce TNF: ${ }^{12,13}$ Grau and others showed higher expression of mRNA of TNF in the spleen macrophages of mice developing cerebral malaria than in those of mice without this complication, and predicted that activation of macrophages by $\mathrm{T}$ cell-released cytokines led to TNF overproduction. ${ }^{14,15}$ In humans, however, this sort of experiment is not appropriate because of ethical and technical considerations in collecting spleen macrophages from patients. So far, TNF concentration has only been measured in peripheral serum samples of $\boldsymbol{P}$. falciparum- and $\boldsymbol{P}$. vivaxinfected patients, and TNF production in the presence of $P$. falciparum components has only been detected using fresh monocytes in culture. 2.9.10.16 Therefore, the relationship of TNF production by mature and activated tissue macrophages to phagocytosis remains to be explored.

In the present work, peripheral monocytes were allowed to mature to macrophages by in vitro cultivation on autologous serum (macrophages) and activated with interferon- $\gamma$ (IFN- $\gamma$ ) (activated macrophages). Production of TNF by macrophages and activated macrophages were examined in relation to their phagocytosis in the presence of various components of $P$. falciparum-infected erythrocytes.

\section{MATERIALS AND METHODS}

Parasites. The FCR-3 strain of $P$. falciparum, kindly donated by Professor M. Suzuki (Gunma University School of Medicine, Maebashi, Japan), was cultured according to the method of Trager and Jensen ${ }^{17}$ in group $O$ erythrocytes ad- justed to 5\% hematocrit in RPMI 1640 medium supplemented with $10 \%$ group $O$ human serum in a moist atmosphere of $5 \% \mathrm{O}_{2}, 5 \% \mathrm{CO}_{2}$, and $90 \% \mathrm{~N}_{2}$. Three separate cultures were synchronized with the sorbitol method at intervals of approximately $12 \mathrm{hr}$ to prepare simultaneously three distinct stages of $\boldsymbol{P}$. falciparum cultures. ${ }^{18}$ Each culture was synchronized three additional times at intervals of approximately $48 \mathrm{hr}$ to narrow the stage variation. The $\mathrm{R}$ stage culture obtained after $12 \mathrm{hr}$ of the final synchronization was composed mostly of ring forms. The $\mathrm{T}$ stage culture obtained after 24-30 hr was mostly trophozoites, and the S stage culture obtained after $36-48 \mathrm{hr}$ was composed of schizonts and new ring forms (Figure 1).

Preparation of monocytes and their maturation and activation. Heparinized venous blood obtained from a healthy Japanese adult male was mixed with an equal volume of phosphate-buffered saline (PBS), and $10 \mathrm{ml}$ of the mixture was gently layered on $3 \mathrm{ml}$ of Histopaque -1077 (Sigma, St. Louis, MO) in a centrifuge tube and centrifuged at $900 \times g$ for $30 \mathrm{~min}$. Mononuclear cells were recovered from the intermediate layer between the plasma and the Histopaque, washed with PBS three times, suspended in RPMI 1640 medium supplemented with $10 \%$ autologous serum at a concentration of $4 \times 10^{6} / \mathrm{ml}$, and $500 \mu \mathrm{l}$ of the suspension was poured into each well of a 24-well cell culture plate in which a 15-mm, round, plastic coverslip (Thermanox; Nunc, Inc., Roskilde, Denamrk) had been placed previously. After incubation for $2 \mathrm{hr}$ at $37^{\circ} \mathrm{C}$ in the $5 \% \mathrm{CO}_{2}$ atmosphere, the plastic coverslip was removed and rinsed in PBS to remove nonadherent cells. The coverslip was then placed in a well of a separate but same type of plate containing $500 \mu l$ of RPMI 1640 medium and used immediately as a source of fresh monocytes. Some of the fresh monocytes were cultured in RPMI 1640 medium with $10 \%$ autologous serum for three days. Thereafter, one portion was cultured in the same fresh medium for three successive days and used as mature monocytes or nonactivated macrophages (hereafter referred to as 


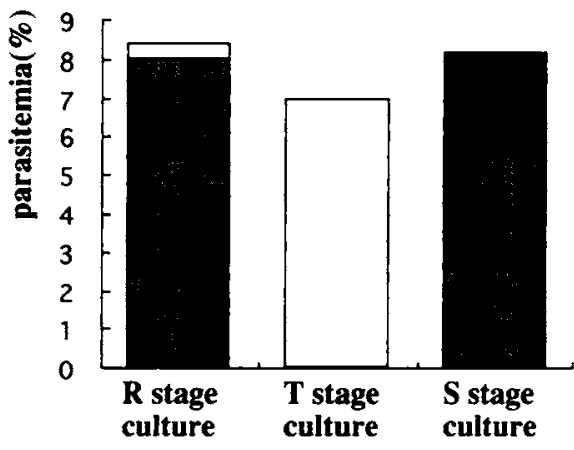

FiguRE 1. Parasitemia and composition of three different stage cultures of Plasmodium falciparum used for tumor necrosis factor induction by fresh monocytes. The $R$ stage cultures were obtained after $12 \mathrm{hr}$ of the final synchronization and consisted of approximately 95\% ring forms (striped bar). The $\mathrm{T}$ stage cultures were obtained after 24-30 hr of synchronization and consisted of exclusively trophozoites (open bar). The $S$ stage cultures were obtained after $36-48 \mathrm{hr}$ of synchronization and consisted of approximately $30 \%$ mature schizonts (stippled bar) and 70\% ring forms (striped bar).

macrophages), ${ }^{19}$ and other portion was cultured in the same medium but supplemented with 300 units $/ \mathrm{ml}$ of IFN- $\gamma$ for the same period and used as activated macrophages.

Preparation of soluble and insoluble components from P. falciparum-infected erythrocytes. Schizont-infected erythrocytes with a parasitemia of $4-5 \%$ were centrifuged at $800 \times g$ for $6 \mathrm{~min}$. The packed cells were measured by volume, lysed by freeze-thawing three times, and centrifuged at $6,000 \times g$ for $4 \mathrm{~min}$. The supernatant was passed through a membrane filter with pore size of $0.2 \mu \mathrm{m}$ and used as soluble components. The precipitate was washed with PBS three times by the same centrifugation, suspended in RPMI 1640 medium to adjust the final volume to that of the original packed cells, and used as insoluble components.

Production of TNF and phagocytosis by monocytes and macrophages in the presence of different stages of $\boldsymbol{P}$. falciparum cultures. Three different stages of $P$. falciparum cultures were centrifuged at $800 \times g$ for $6 \mathrm{~min}$ and $30 \mu \mathrm{l}$ of packed infected erythrocytes were added to each well with fresh monocytes, macrophages, or activated macrophages in $500 \mu \mathrm{l}$ of RPMI 1640 medium and incubated for $5 \mathrm{hr}$ at $37^{\circ} \mathrm{C}$ in a $5 \% \mathrm{CO}_{2}$ atmosphere. An incubation time of $5 \mathrm{hr}$ was chosen to minimize the effect on TNF production by stage development of parasites. After incubation, the overlay medium was centrifuged at $6,000 \times g$ for $10 \mathrm{~min}$ and the supernatant was kept at $-20^{\circ} \mathrm{C}$ until the TNF assay. A plastic coverslip was rinsed in PBS, immersed in distilled water for $20 \mathrm{sec}$ to lyse erythrocytes adhering to monocytes, and rinsed in PBS again. The coverslip was then dried, fixed with methanol, stained with Giemsa, and examined microscopically to evaluate phagocytosis of infected erythrocytes by monocytes. Noninfected erythrocytes were used as a negative control for TNF production and phagocytosis. Lipopolysaccharide (LPS) (from Escherichia coli; Sigma) was used as a positive control for TNF production.

Production of TNF and phagocytosis of monocytes and macrophages in the presence of soluble and insoluble components. Soluble or insoluble components $(30 \mu \mathrm{l})$, prepared as described above, were added to separate culture wells containing monocytes and macrophages. After a 24-hr incubation at $37^{\circ} \mathrm{C}$ in a $5 \% \mathrm{CO}_{2}$ atmosphere, an overlay medium was used for the TNF assay and a plastic coverslip was used for the phagocytosis assay as described below. Noninfected erythrocytes and LPS were used as negative and positive controls, respectively.

Assay for TNF. Tumor necrosis factor concentration was measured by its cytotoxic effect on L-929 murine fibroblast cells (Dainippon Pharmaceutical Co., Osaka, Japan) ${ }^{20}$ Briefly, $100 \mu \mathrm{l}$ of an L-929 cell suspension in RPMI 1640 medium at a concentration of $4 \times 10^{5} / \mathrm{ml}$ was seeded in each well of a microculture plate with 96 wells and incubated at $37^{\circ} \mathrm{C}$ in a $5 \% \mathrm{CO}_{2}$ atmosphere for $24 \mathrm{hr}$. A total of $50 \mu \mathrm{l}$ of RPMI 1640 medium containing $0.4 \mu \mathrm{g}$ of actinomycin D and $50 \mu \mathrm{l}$ of a serial two-fold dilution of the overlay medium was added to each well and incubated for 18-20 hr. As a control, $100 \mu$ l of overlay medium containing actinomycin $D$ with noninfected erythrocytes was used. The wells were then washed with PBS, stained with crystal violet, washed with distilled water, and measured colorimetrically for optical density by an enzyme-linked immunosorbent assay at $590 \mathrm{~nm}$. Cell destruction was expressed as the percentage of the optical density of each well compared with that of the control. One unit of TNF was defined as the amount of TNF causing 50\% cell destruction. Thus, the TNF unit of each overlay medium was expressed as the reciprocal of the dilution that showed $50 \%$ cell destruction.

Assay for phagocytosis. The phagocytosis rate was expressed as the number of monocytes or macrophages that ingested malaria pigment per 200 monocytes or macrophages. In our assay system, monocytes or macrophages ingesting infected erythrocytes or merozoites were rarely seen.

\section{RESULTS}

Production of TNF and phagocytosis by fresh monocytes in the presence of different stages of parasites. As shown in Figure 2, $\mathrm{S}$ stage cultures induced fresh monocytes to release a significantly higher level of TNF than other stage cultures $(P=0.022$, by Kruskal-Wallis test). The $\mathrm{T}$ stage cultures were intermediate in this activity, while $R$ stage cultures showed the lowest level of TNF released. Phagocytosis by fresh monocytes was also highest against $\mathrm{S}$ stage cultures, while phagocytosis against the $R$ stage cultures was slightly higher than against the $T$ stage cultures (Figure 3 ). This may be due to the fact that more free malaria pigment released from infected erythrocytes with mature parasites synchronized by sorbitol treatment remained in the $R$ stage cultures than in $T$ stage cultures. Since $S$ stage cultures prove to be the most effective, they were exclusively used in the following experiments.

Production of TNF and phagocytosis by macrophages. As shown in Figure 4A, little production of TNF by macrophages was seen in the presence of $S$ stage cultures. In addition, TNF production by macrohages in the presence of LPS was also lower compared with that of fresh monocytes although it was higher than in the presence of $\mathbf{S}$ stage cultures. On the other hand, phagocytosis was much more active by macrophages than by fresh monocytes (Figure $4 B$ ), suggesting a maturation process of monocytes enhancing the phagocytosis function. Since TNF production by macro- 


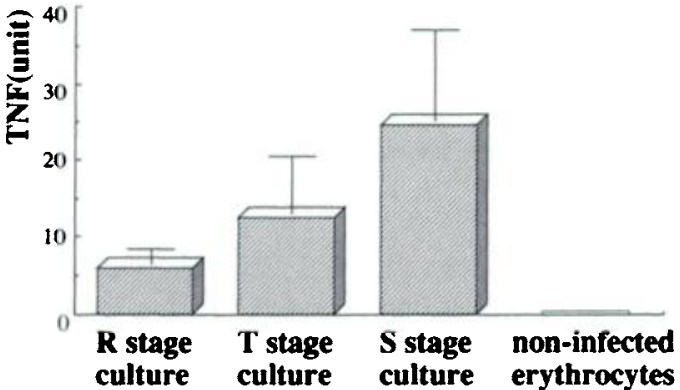

FIGURE 2. Tumor necrosis factor (TNF) production by fresh monocytes after $5 \mathrm{hr}$ incubation in the presence of $R, T$, and $S$ stage cultures of Plasmodium falciparum. The TNF unit was expressed as the reciprocal of the dilution that showed 50$) \%$ cell destruction in the TNF assay. Bars show the mean $\pm \mathrm{SD}$.

phages in the presence of LPS was lower than by fresh monocytes, macrophages were activated by IFN- $\gamma$ and their TNF productivity was examined. As shown in Figure 5. TNF production by activated macrophages did not differ from that by nonactivated macrophages in the presence of $S$ stage cultures, while TNF production by activated macrophages in the presence of LPS increased significantly. Phagocytosis of activated macrophages was similar to that of nonactivated macrophages and much higher than that of fresh monocytes in the presence of S stage cultures.

Monocytes and macrophages phagocytized malaria pigment almost exclusively and rarely phagocytized merozoites or infected erythrocytes. This suggested that malaria pigment was a preferable target for phagocytosis, and that this activity does not require antibodies.

Effects of soluble and insoluble components of $P$. falciparum-infected erythrocytes on TNF production and phagocytosis by monocytes or macrophages. To clarify potential TNF-inducing factors from $S$ stage cultures and the relationship between TNF production and phagocytosis, soluble and insoluble components were added separately to a culture well containing fresh monocytes or activated macrophages. Fresh monocytes produced TNF in the presence of both soluble and insoluble components (Figure 6). This indicated that not only soluble components, but also insoluble components, contained TNF-inducing factors for monocytes. The phagocytosis of fresh monocytes and activated macrophages in the presence of insoluble components is

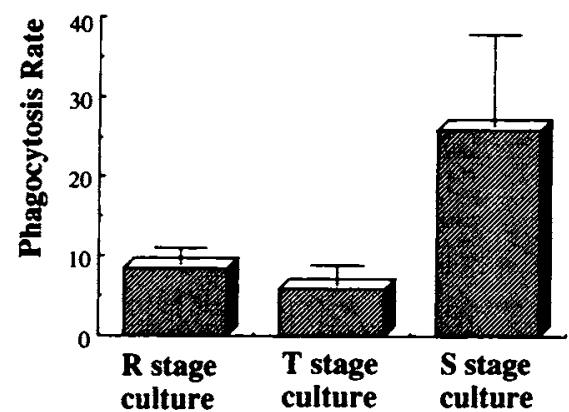

FigURE 3. Phagocytosis rate of malaria pigment by fresh monocytes in the presence of three different stage cultures of Plasmodium falciparum. The phagocytosis rate was expressed as the number of monocytes containing malaria pigment per 200 monocytes. Bars show the mean $\pm S D$.

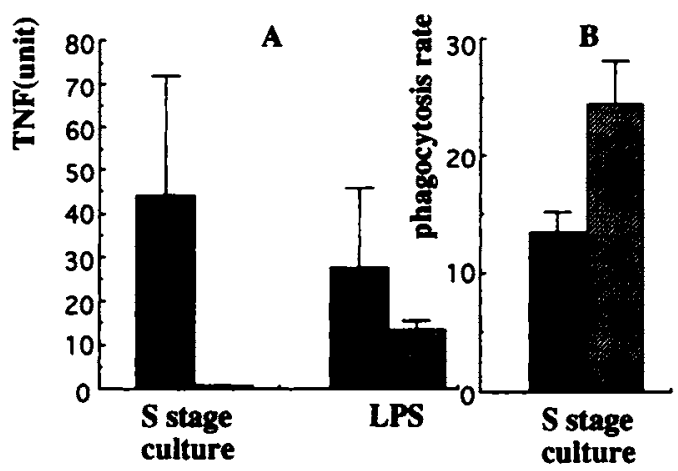

FIGURE 4. Comparison of A, tumor necrosis factor (TNF) production and B, phagocytosis between fresh monocytes (dark bars) and nonactivated macrophages (mature monocytes) (striped bars) in the presence of $S$ stage cultures of Plasmodium falciparum or lipopolysaccaride (LPS). Bars show the mean \pm SD.

shown in Figure 7A and B, respectively. Since monocytes or macrophages came into more frequent and longer contact with malarial pigment in the presence of insoluble components than in the presence of $S$ stage cultures, they phagocytized much more malarial pigment under the former conditions. Larger quantities of phagocytized pigment were also observed in macrophages than in monocytes. The quantity of ingested pigment in IFN- $\gamma$-activated macrophages was similar to that in nonactivated macrophages. The production of TNF by activated macrophages in the presence of soluble and insoluble components was also extremely low (Figure 6).

\section{DISCUSSION}

Tumor necrosis factor, which is produced mainly by macrophages, is almost always related to immunopathologic reactions in microbial infection. ${ }^{21}$ Recently, the implication of TNF in the pathogenesis of malaria has been recognized. 1, 2, 9-11 The mechanism of TNF induction in Plasmodium infection has been studied extensively and soluble substances that were released from infected erythrocytes at schizont rupture were shown to be the most probable TNF-

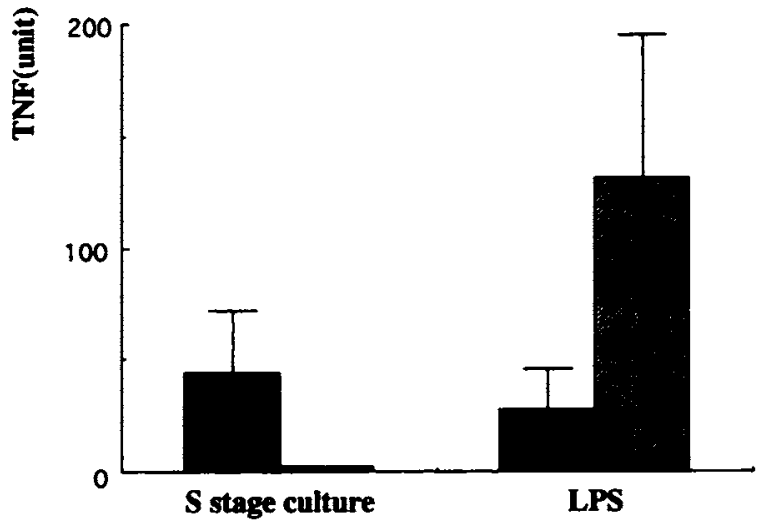

Figure 5. Comparison of tumor necrosis factor (TNF) production between fresh monocytes (dark bars) and activated macrophages (striped bars) in the presence of $S$ stage cultures of Plasmodium falciparum or lipopolysaccharide (LPS). Bars show the mean \pm SD. 


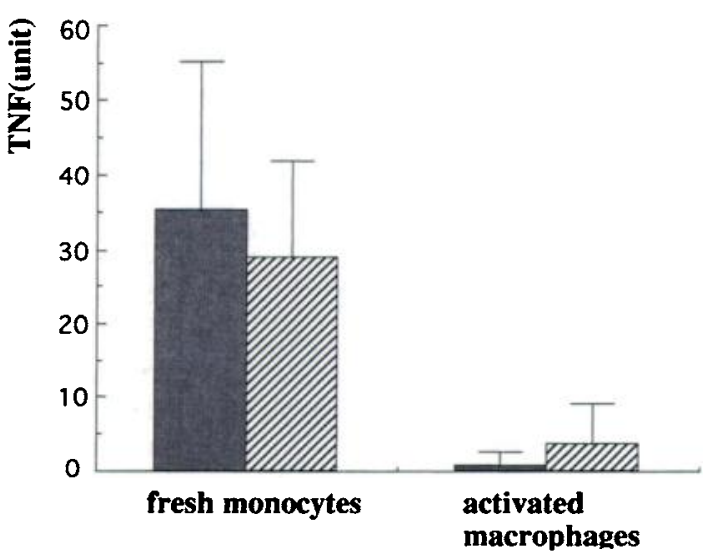

FIGURE 6. Tumor necrosis factor (TNF) production by fresh monocytes and activated macrophages in the presence of soluble (dark bars) or insoluble components (striped bars) of Plasmodium falciparum-infected erythrocytes. Bars show the mean \pm SD.

inducing factors for macrophages, ${ }^{7.12 .22 .23}$ although other candidates were also determined. ${ }^{24}$ The present result also showed that $\mathrm{S}$ stage cultures induced the highest production of TNF by monocytes.

The most interesting finding in the present work was that matured macrophages and even IFN- $\gamma$-activated macrophages in vitro produced little TNF in the presence of the $S$ stage culture of the parasite, whereas they developed phagocytic activity and TNF productivity in the presence of LPS. The finding that soluble and insoluble components from infected erythrocytes almost equally stimulated monocytes to produce TNF suggested that phagocytosis was not related to TNF production because only the latter components were phagocytized vigorously. Our results indicated that mono- cytes were stimulated via direct contact by both soluble and insoluble components and that if they recognized stimulants by receptors on the cell surface as in the case of LPS, ${ }^{25}$ they might lose them or become unable to trigger TNF production even after recognition during the maturation process to macrophages. Recently, malarial pigment were shown to be more active as TNF-inducing factors for fresh monocytes, irrespective of phagocytosis, than soluble components. ${ }^{24}$ These results suggested the presence of several TNF-inducing factors and provided evidence against the proposal of an antidisease vaccine aimed at inhibition of TNF production by specific antibodies.

It is not known whether matured monocytes in vitro correspond to tissue macrophages. If that is the case, it is thought that TNF production occurs mainly in the blood stream, but not in tissues rich in fixed or migrating macrophages, suggesting a low possibility of TNF accumulation in local lesions. In fact, there was no evidence that monocytes had migrated to the region where infected erythrocytes were sequestered. ${ }^{26}$ Although the specific immunity to malaria parasites is thought to play the major role in clinical immunity, these findings partially explain the fact that malaria symptoms become milder after frequent infections when macrophage activity is supposed to be increased. In addition, a big disparity in phagocytosis between monocytes and macrophages seems to be correlated with the fact that malaria pigment is almost exclusively seen in reticuloendothelial tissues rich in fixed macrophages such as the spleen and the liver.

In mouse models, peritoneal macrophages treated with IFN- $\gamma$ produced larger amounts of TNF than untreated ones, and higher TNF production was shown in the mice that developed cerebral malaria compared with mice that did not

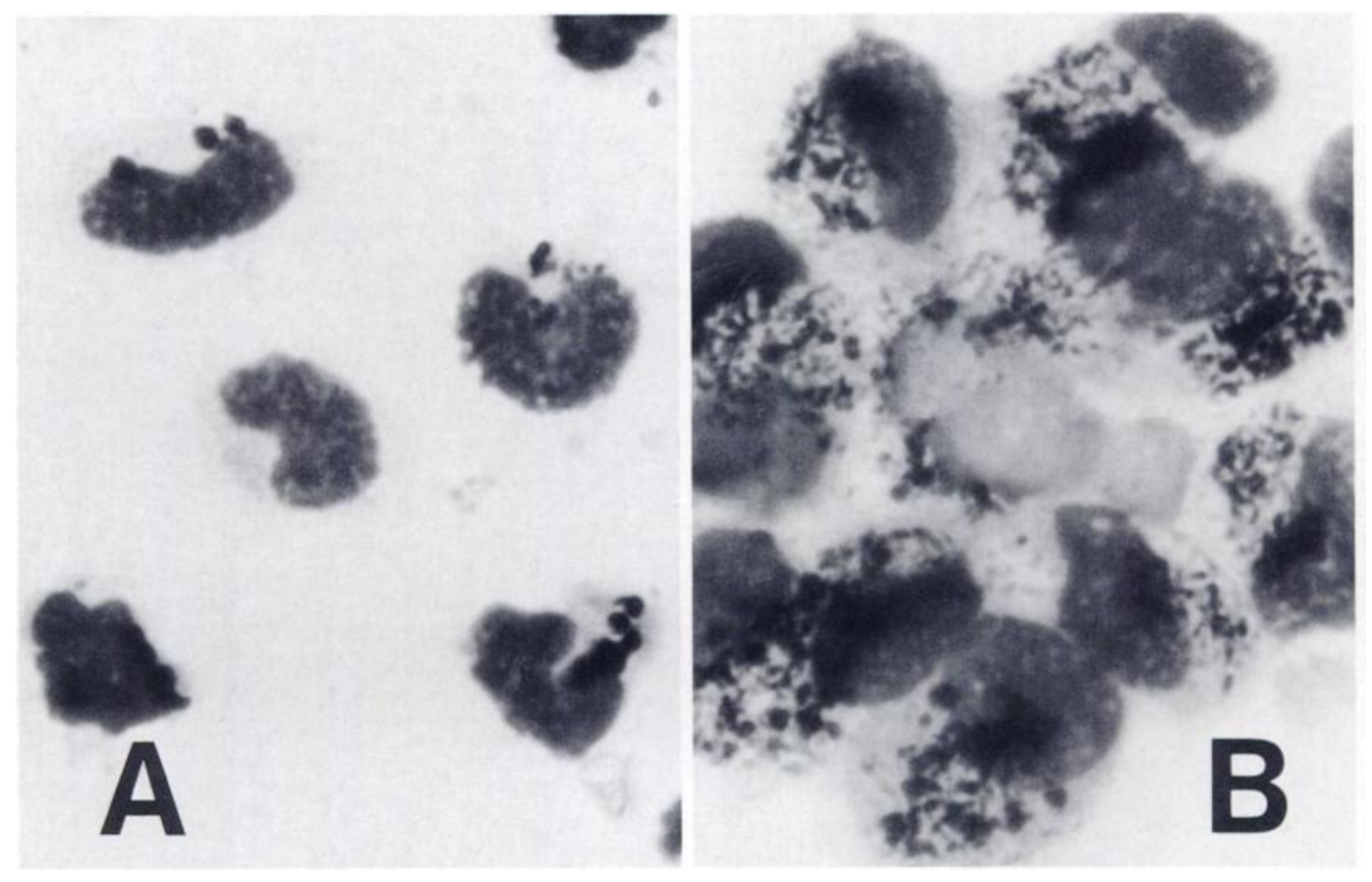

FIGURE 7. Phagocytosis of insoluble components by A, fresh monocytes and B, activated macrophages. A much higher phagocytosis rate and much larger quantity of ingested malaria pigment were seen in activated macrophages (Giemsa stained, magnification $\times 1,000$ ). 
develop such complications. ${ }^{14.15}$ These results in the mouse model appear to be quite different from our postulate concerning human malaria. In the present work, however, we did not consider the in vivo condition in which complex cytokine networks are involved, resulting in a variety of TNF-producing mechanisms. Furthermore, we used only a few individual animals; thus, we must take individual genetic differences into consideration. It is plausible that monocytes from some genetic groups of individuals maintain or increase TNF production even after their maturation to macrophages, causing severe malaria as shown in the mouse model. In addition, we must also consider the strain differences of $P$. falciparum among which TNF induction by different isolates showed a wide variation, ${ }^{24.27}$ indicating the possibility that some strains could stimulate macrophages as well as monocytes to produce TNF. These speculations should be clarified by studies using various $P$. falciparum isolates and macrophages from individuals with different genetic backgrounds.

Although we used only one strain and a few donors of monocytes, our present findings support the important suggestion that monocytes in the blood stream play the major role in TNF production in $P$. falciparum malaria and consequently, the suspected higher concentration of TNF in local lesions might not be accurate.

Acknowledgments: We thank Shusuke Nakazawa for kindly commenting on the manuscript and Miki Kinoshita for preparing the manuscript.

Authors' addresses: Takayoshi Nagao, Haruki Uemura, Tetsuo Yanagi, and Hiroji Kanbara, Department of Protozoology, Institute of Tropical Medicine, Nagasaki University, 1-12-4 Sakamoto, Nagasaki 852. Japan. Kazunori Oishi and Tsuyoshi Nagatake, Department of Internal Medicine, Institute of Tropical Medicine, Nagasaki University, 1-12-4 Sakamoro, Nagasaki 852, Japan.

Reprint requests: Hiroji Kanbara, Department of Protozoology, Institute of Tropical Medicine, Nagasaki University, 1-12-4 Sakamoto, Nagasaki 852, Japan.

\section{REFERENCES}

1. Scuderi P, Sterling KE, Finley PR, Ryan KJ, Ray CG, 1986. Raised serum levels of tumor necrosis factor in parasitic infections. Lancet ii: 1364-1365.

2. Karunaweera ND, Grau GE, Gamage P, Carter R, Mendis KN, 1992. Dynamics of fever and serum levels of tumor necrosis factor are closely associated during clinical paroxysms in Plasmodium vivax malaria. Proc Natl Acad Sci USA 89: 3200-3203.

3. Clark IA, Hunt NH, Butcher GA, Cowden WB, 1987. Inhibition of murine malaria (Plasmodium chabaudi) in vivo by recombinant interferon-gamma or tumor necrosis factor, and its enhancement by butylated hydroxyanisole. J Immunol 139: 3493-3496.

4. Rook GAW, 1991. Evaluation of TNF as an antiviral, antibacterial and antiparasitic agent. Biotherapy 3: 167-175.

5. Taverne J, Matthews N, Depledge P, Playfair JHL, 1984. Malarial parasites and tumour cells are killed by the same component of tumour necrosis serum. Clin Exp Immunol 57: 293300.

6. Wozencraft AO, Dockrell HM, Taverne J, Targett GAT, Playfair JHL, 1984. Killing of human malaria parasites by macrophage secretory products. Infect Immun 43: 664-669.
7. Bate CA, Taverne J, Playfair JH, 1989. Soluble malarial antigens are toxic and induce the production of tumour necrosis factor in vivo. Immunology 66: 600-605.

8. Grau GE, Fajardo LF, Piguet PF, Allet B, Lambert PH, Vassalli $P, 1987$. Tumor necrosis factor (cachectin) as an essential mediator in murine cerebral malaria. Science 237: 1210-1212.

9. Grau GE, Taylor TE, Molyneux ME, Wirima JJ, Vassalli P, Hornmel M, Lambert PH, 1989. Tumor necrosis factor and disease severity in children with falciparum malaria. $N$ Engl $J$ Med 320: 1586-1591.

10. Kwiatkowski D, Hill AV, Sambou I, Twumasi P, Castracane J, Manogue KR, Cerami A, Brewster DR, Greenwood BM, 1990. TNF concentration in fatal cerebral, non-fatal cerebral, and uncomplicated Plasmodium falciparum malaria. Lancet 336: 1201-1204.

11. Shaffer N, Grau GE, Hedberg K, Davachi F, Lyamba B, Hightower AW, Breman JG, Phuc ND, 1991. Tumor necrosis factor and severe malaria. J Infect Dis 163: 96-101.

12. Bate CA, Taverne J, Roman E, Moreno C, Playfair JH, 1992. Tumour necrosis factor induction by malaria exoantigens depends upon phospholipid. Immunology 75: 129-135.

13. Kwiatkowski D, Cannon JG, Manogue KR, Cerami A, Dinarello CA, Greenwood BM, 1989. Tumour necrosis factor production in falciparum malaria and its association with schizont rupture. Clin Exp Immunol 77: 361-366.

14. Grau CE, Piguet PF, Lambert PH, 1992. Immunopathology of malaria: role of cytokine production and adhesion molecules. Mem Inst Oswaldo Cruz 87 (suppl V): 95-100.

15. Grau GE, Piguet PF, Vassalli P, Lambert PH, 1989. Tumor necrosis factor and other cytokines in cerebral malaria: experimental and clinical data. Immunol Rev 112: 49-70.

16. Picot S, Peyron F, Vuillez J-P, Barbe G, Marsh K. AmbroiseThomas $P, 1990$. Tumor necrosis factor production by human macrophages stimulated in vitro by Plasmodium falciparum. Infect Immun 58: 214-216.

17. Trager W, Jensen JB, 1976. Human malaria parasites in continuous culture. Science 193: 673-675.

18. Lambros C, Vanderberg JP, 1979. Synchronization of Plasmodium falciparum erythrocytic stages in culture. $J$ Parasitol 65: 418-420.

19. Musson RA, 1983. Human serum induces maturation of human monocytes in vitro. Am Assoc Pathologists 111: 331-340.

20. Ruff MR, Gifford GE, 1980. Purification and physico-chemical characterization of rabbit tumor necrosis factor. $J$ Immunol 125: $1671-1677$.

21. Tracey KJ, Vlassara H, Cerami A, 1989. Cachectin/tumor necrosis factor. Lancet $i$ : $1122-1126$.

22. Bate CA, Taverne J, Bootsma HJ, Mason RC, Skalko N, Gregoriadis G, Playfair JH, 1992. Antibodies against phosphatidylinositol and inositol monophosphate specifically inhibit tumour necrosis factor induction by malaria exoantigens. Immunology 76: 35-41.

23. Bate CA, Taverne J, Playfair JH, 1992. Detoxified exoantigens and phosphatidylinositol derivatives inhibit tumor necrosis factor induction by malarial exoantigens. Infect Immun 60: 1894-1901.

24. Pichyangkul S, Saengkrai P, Webster HK, 1994. Plasmodium falciparum pigment induces monocytes to release high levels of tumor necrosis factor- $\alpha$ and interleukin-1 $\beta$. Am J Trop Med Hyg 51: 430-435.

25. Wright SD, Ramos RA, Tobias PS, Ulevitch RJ, Mathison JC, 1990. $\mathrm{CD}_{14}$, a receptor for complexes of lipopolysaccharide (LPS) and LPS binding protein. Science 249: 1431-1433.

26. Aikawa M, Pongponratn E, Tegoshi T, Nakamura $K$, Nagatake T, Cochrane A, Ozaki LS, 1992. A study on the pathogenesis of human cerebral malaria and cerebral babesiosis. Mem Inst Oswaldo Cruz 87 (suppl III): 297-301.

27. Allan RJ, Rowe A, Kwiatkowski D, 1993. Plasmodium falciparum varies in its ability to induce tumor necrosis factor. Infect Immun 61: 4772-4776. 